NIST Technical Note 2085

\title{
Workshop on Smart Grid Interoperability Testing and Certification
}

\author{
Cuong Nguyen \\ Avi Gopstein
}

This publication is available free of charge from:

https://doi.org/10.6028/NIST.TN.2085 


\title{
Workshop on Smart Grid Interoperability Testing and Certification
}

\author{
Cuong Nguyen \\ Avi Gopstein \\ Smart Grid and Cyber-Physical Systems Program Office \\ Engineering Laboratory
}

This publication is available free of charge from:

https://doi.org/10.6028/NIST.TN.2085

January 2020

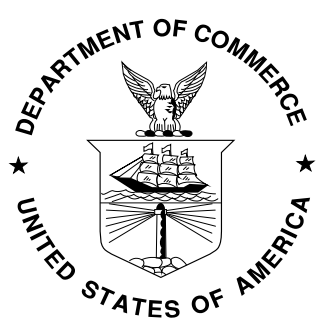

U.S. Department of Commerce Wilbur L. Ross, Jr., Secretary

National Institute of Standards and Technology Walter Copan, NIST Director and Undersecretary of Commerce for Standards and Technology 
Certain commercial entities, equipment, or materials may be identified in this document in order to describe an experimental procedure or concept adequately. Such identification is not intended to imply recommendation or endorsement by the National Institute of Standards and Technology, nor is it intended to imply that the entities, materials, or equipment are necessarily the best available for the purpose.

National Institute of Standards and Technology Technical Note 2085

Natl. Inst. Stand. Technol. Tech. Note 2085, 33 pages (January 2020) CODEN: NTNOEF

This publication is available free of charge from:

https://doi.org/10.6028/NIST.TN.2085 


\begin{abstract}
New smart grid architectures, devices, systems, and applications require extensive data exchange, necessitating well-defined interfaces to transfer and translate this data across the grid. Clarifying the protocols and data performance requirements across channels of communication is critical to easing the integration and interoperability of smart grid technologies. Testing and certification (T\&C) programs provide a mechanism for all stakeholders to gain confidence that equipment will operate as intended and conforms to standards. The added confidence afforded by T\&C strengthens the standards ecosystem and fosters technology innovation and adoption. Companies can also advertise that products or services conform with a recognized technical standard or specification. While T\&C programs do exist for smart grid standards, they are currently limited in number and in general are focused on a subset of metrics that cannot in isolation guarantee interoperability.

This report presents the results of a workshop co-hosted by NIST with the Smart Electric Power Alliance and the Institute of Electrical and Electronics Engineers (IEEE) Standards Association in Washington, DC. The purpose was to explore underlying drivers for the current state of T\&C programs for smart grid interoperability. The workshop also examined interoperability profiles for smart grid standards as a means to accelerate the development of T\&C programs. Workshop attendees included nearly 50 experts from the smart grid stakeholder community, including representatives from industry, academia, government, professional associations, and testing and certification bodies.
\end{abstract}

\title{
Keywords
}

Cyber-physical systems, internet of things, interoperability, smart grid, testing and certification. 


\section{Table of Contents}

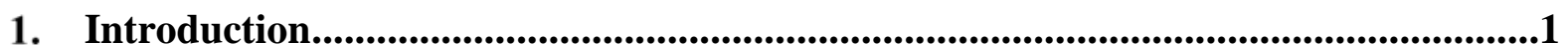

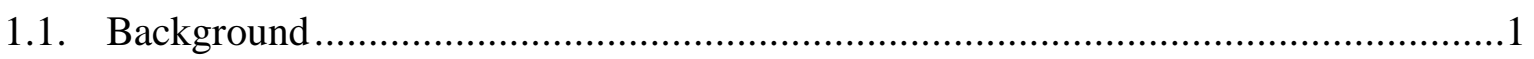

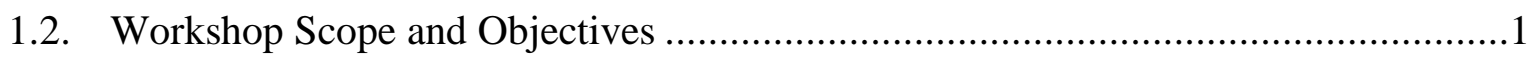

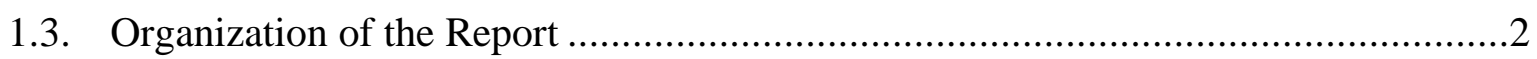

2. Keynote and Panels: Value Proposition for Testing and Certification .........................2

2.1. Keynote: Vision and Role for Smart Grid Testing and Certification...........................3

2.2. Panel: Value Proposition for Testing \& Certification (T\&C) .................................... 4

2.2.1. Panelist Highlights.........................................................................................

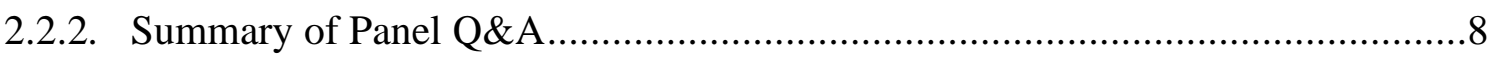

3. Smart Grid Interoperability T\&C Challenges................................................................9

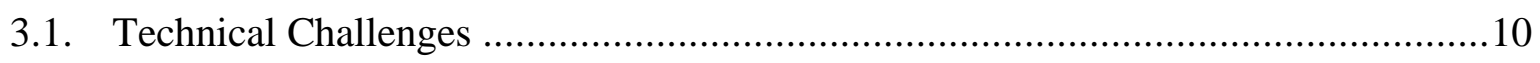

3.2. Non-Technical Challenges...............................................................................12

4. Smart Grid Interoperability Profile Elements .................................................................14

4.1. Profile Elements for Physical Assets..................................................................15

4.2. Profile Elements for Communication Protocols .....................................................16

4.3. Profile Elements for Information Models.............................................................17

5. Approaches for Developing T\&C Programs for Interoperability ..............................18

5.1. Potential Pathways to Buy-in and Acceptance of Interoperability T\&C ...................18

5.2. Design Approaches for Interoperability T\&C ......................................................20

6. Smart Grid Interoperability Scenarios Priority Interfaces ..........................................20

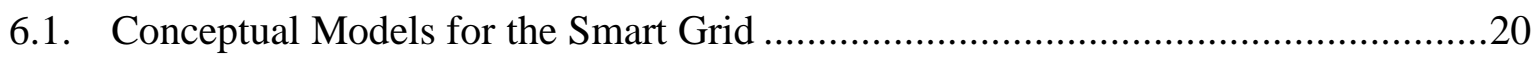

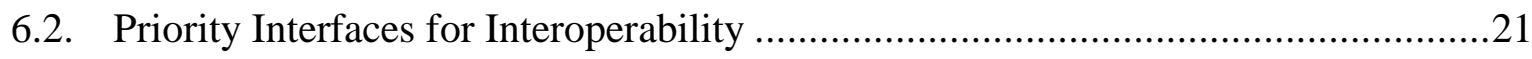

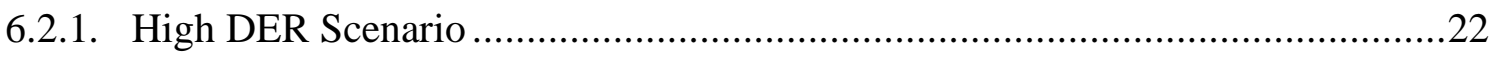

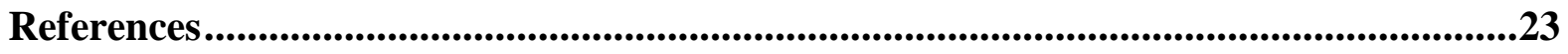

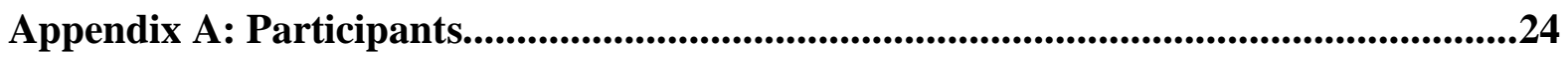

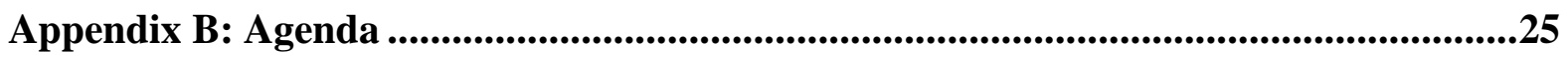

Appendix C: Background Materials..................................................................................26

Appendix D: Acronyms.............................................................................................................27

\section{List of Tables}

Table 3.1 Technical Challenges for SG Interoperability T\&C Programs.......................11

Table 3.2 Non-Technical Challenges for SG Interoperability T\&C Programs................13

Table 4.1 Smart Grid Interoperability Profile Elements for Physical Assets ..................16 
Table 4.2. Smart Grid Interoperability Profile Elements for Communication Protocols

Table 4.3. Smart Grid Interoperability Profile Elements for Information Models ........18

Table 5.1. Smart Grid Interoperability T\&C Approaches ...........................................20

Table 6.1. Smart Grid Interoperability Priority Interfaces for High DER Architecture

Scenario .22

\section{List of Figures}

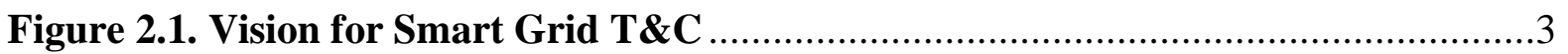

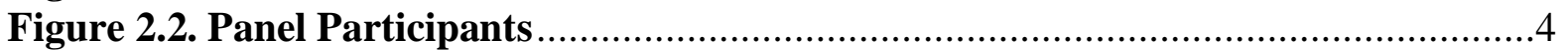

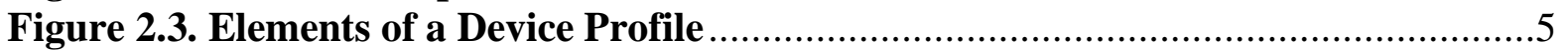

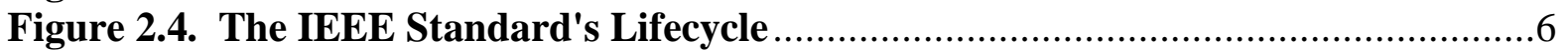

Figure 2.5. Multi-Speak Ecosystem ………..........................................................

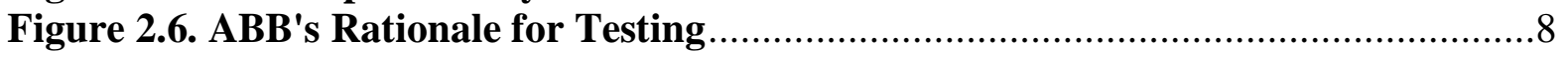

Figure 3.1. Focus Questions for Challenges.............................................................10

Figure 4.1 Focus Questions for Interoperability Profiles..............................................14

Figure 5.1. Focus Questions for T\&C Program Approaches.......................................18

Figure 5.2. Business Models of Utility T\&C Programs...............................................2

Figure 6.1. Focus Questions for Priority Interfaces ...................................................21 


\section{Introduction}

\subsection{Background}

New smart grid architectures, devices, systems, and applications require extensive data exchange, necessitating well-defined interfaces to transfer and translate this data across the grid. Interoperability is a critical enabler of many smart grid benefits and is key to reducing system integration costs as new capabilities are deployed.

Smart grid standards play a key role in the adoption of new technologies for the power grid. Standardization establishes consistent, universally understood protocols and guidelines that-if adopted-enable interoperability and compatibility of equipment regardless of vintage or manufacturer. Testing and certification (T\&C) programs are a mechanism for stakeholders to gain confidence that equipment function conforms to the appropriate standards and will operate as intended. The added confidence afforded by T\&C fosters technology acceptance and strengthens the standards ecosystem. Companies providing products or services that conform with a recognized technical standard or specification can also reach a broader customer base.

The standards landscape for smart grid interoperability is complex and spans many dimensions and domains of the grid, with T\&C achieved through either first party (selfcertification), second party (user), or third party (independent certification) testing. NIST examined T\&C program accessibility for smart grid interoperability standards, and found their availability lacking [1].

\subsection{Workshop Scope and Objectives}

On July 9, 2018, NIST co-hosted a workshop with the Smart Electric Power Alliance and the Institute of Electrical and Electronics Engineers (IEEE) Standards Association in Washington, D.C. to explore underlying drivers for the current state of T\&C for smart grid interoperability. The workshop also examined interoperability profiles for smart grid standards as a means to accelerate the development of T\&C programs. Workshop attendees included nearly 50 experts from the smart grid community, including stakeholders from industry, academia, government, professional associations, and T\&C bodies. A full list of attendees is included in Appendix A.

The workshop included a combination of plenary speakers, panels, and breakout sessions (see Appendix B, Agenda). Breakout sessions were focused on three topics that encompass the primary elements necessary to create an interoperability profile. During these sessions, participants provided feedback on the essential elements for T\&C programs related to interoperability standards, as well as some of the challenges to creating and implementing these programs.

NIST introduced participants to a set of preliminary diagrams included in a draft update to the NIST Framework and Roadmap for Smart Grid Interoperability Standards, Release 3.0 (Framework) [2] to illustrate various communications pathways scenarios that explore 
evolving device and network interfaces through architecturally driven interpretations of the Smart Grid Conceptual Model. These scenarios include:

- Legacy Utility Architecture

- Hybrid Architecture

- Microgrid Architecture

- High-Distributed Energy Resource (DER) Architecture

Participants provided general perspectives and feedback on these diagrams, including for example, comments on new interactions, scales, domains, mappings or gaps. Insights were gained on what participants considered to be some of the priority interfaces in these scenarios, i.e., where interoperability will play a critical role in the modern and evolving grid. In advance of the workshop, read-ahead materials were provided to inform the participants about the topics to be discussed and to stimulate discussions. Appendix $\mathrm{C}$ provides links to these materials as well as the plenary presentations.

\subsection{Organization of the Report}

This document is a factual reporting of the discussions that took place during this workshop. The report includes chapters organized according to the plenary, panel, and topical sessions conducted, including:

1) Value proposition for T\&C programs;

2) Drivers and challenges to developing and implementing $T \& C$ programs for interoperability standards;

3) Priority profile elements for interoperability ; and

4) Priority interfaces for interoperability in proposed grid architectural approaches.

During the workshop, participants were split into breakout groups that worked on the same set of questions, but in their separate groups. Each breakout group included a range of experts with different skill sets and demographics (industry, academia, government, etc.). The compiled responses from each group form the basis for the topical chapters in this report. The results presented here are not all-inclusive of the smart grid community. They do represent a snapshot of perspectives based on those who attended and participated in discussions. Registered attendees included a range of individuals with diverse backgrounds and experiences, and with strong expertise in the topics of interest at the workshop.

\section{Keynote and Panels: Value Proposition for Testing and Certification}

In addition to welcoming remarks by NIST organizers, a keynote presentation and moderated panel set the stage for topical discussions around T\&C programs and other key topics. The following summary provides highlights of the remarks as well as question and answer sessions. 


\subsection{Keynote: Vision and Role for Smart Grid Testing and Certification}

Jason Handley, Duke Energy, presented his vision (Figure 2.1) for deploying systems for smart grids with greater interoperability, and gave perspectives on the important role of T\&C in grid modernization. There are multiple testing and certification entities serving a variety of roles, including Interoperability Testing and Certification Authorities, standards development organizations, test laboratories, certification bodies, and accreditation bodies. The benefits of certification are many. It provides certainty in markets for customers, implementers, and product vendors and increases confidence in the interoperability of implemented projects.

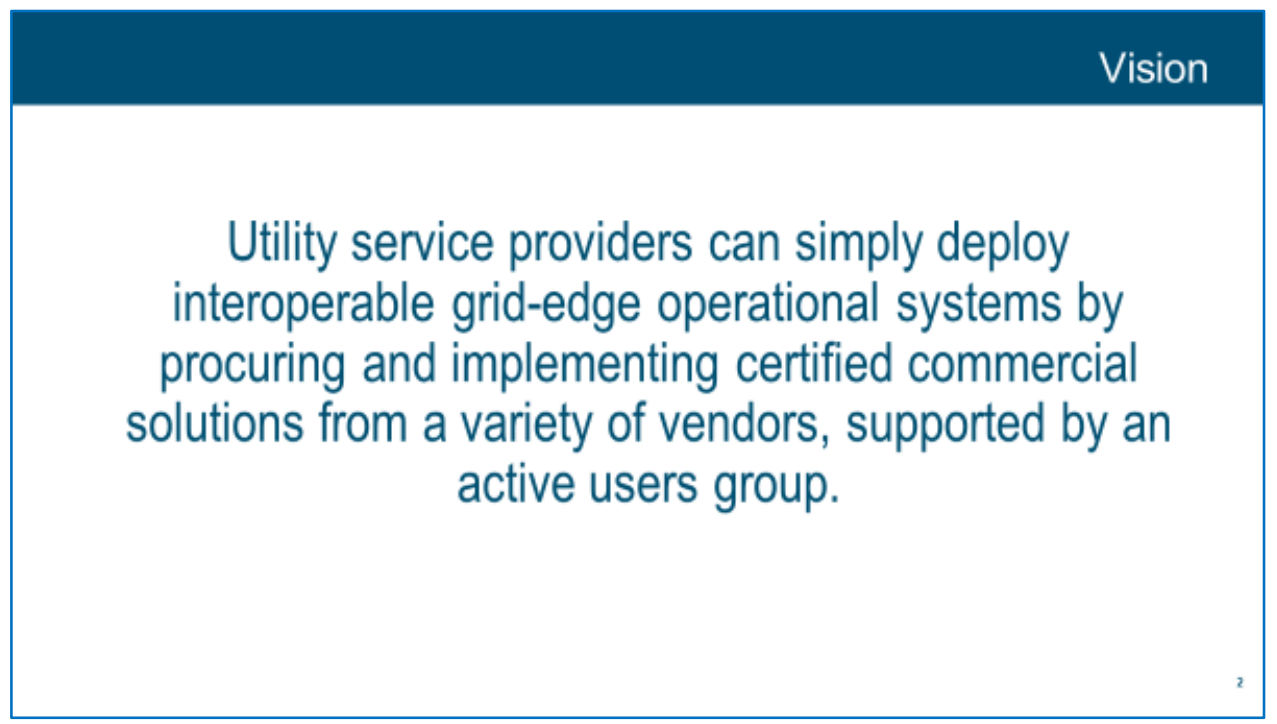

\section{Figure 2.1. Vision for Smart Grid T\&C}

Utilities need more interoperable systems, but often the utilities are part of the problem because their specifications have led to non-interoperability in their system or with current standards and commercial products. Duke Energy seeks to integrate the "best of breed" in all equipment but cannot because they do not work together. As a potential solution, Duke Energy is starting to use open-source implementations more and more to address interoperability challenges by leveraging community knowledge and experience.

Certification of interoperability would increase value by establishing thresholds and clarifying the difference between asserting conformance to a standard and having interoperability. Compliance is relatively familiar and well managed; conformance is more difficult to ascertain, and a current focus of efforts at Duke Energy. The distinction between compliance and conformance is the former implies the vendor's support for the standard in the design of the product, while the latter validates the product implementation meets all of the requirements in the standard. Duke Energy cannot get to interoperability between systems without a massive amount of back-office integration of the head-end systems, instead of using data where it is most valuable which is often at the periphery of their system. In their case, Duke Energy's interoperability only exists at their data centers. Duke Energy spends about $\$ 140$ million per year on back-office integration of data from non-interoperable systems. 
To be effective, T\&C should integrate many entities and processes (supply chain, safety, reliability, interoperability, security, standardization, and communications).

Communications are the backbone of any Smart Grid program; reliable, fast communications are necessary for performance. Standardization prevents one-off designs and solutions in functionality that have reached commodity status; a goal is to bring utilities to commodity scale. Conformance always produces a safer, more reliable product.

Open Field Message Bus (OpenFMB) is an example process for conformance and certification. OpenFMB is "a framework and reference architecture comprised of existing standards that enables grid edge interoperability and distributed intelligence, augments operational systems, and enhances integration with field devices.” OpenFMB nodes make it possible to have local data securely available between assets at the grid edge rather than going to a central system, increasing situational awareness and the ability to affect local control in coordination with other operational decision-making [3]. Computing has changed so much over five years; memory and computation are cheaper, allowing us to do more computing at the edge.

Duke Energy is concerned about the security of the Internet Protocol (IP) routable equipment going on the system. Another concern is the proliferation of end devices such as DER. Duke Energy has a very modern advanced distribution management system (ADMS) but it was installed to only handle one million nodes. In the last 5 years, Duke Energy has connected 600,000 nodes, and that is only utility-owned DER devices. The system will not be able to handle future expansions.

Currently, there is a lack of continued momentum in developing testing programs to support the smart grid. Utilities can do more in this arena. Writing standards, T\&C and minimum performance levels into the procurement process is a good approach. Obtaining buy-in from supply chain and product line managers is also critical.

\subsection{Panel: Value Proposition for Testing \& Certification (T\&C)}

Understanding and raising awareness of the value proposition and benefits of T\&C is key to fostering development and acceptance of T\&C programs for interoperability. As summarized below, the moderated panel discussed real-world examples of T\&C programs and the benefits to product developers and end-users. Panel participants are listed in Figure 2.2.

Panel Participants

Moderator Bill Colavecchio, Underwriters Laboratory

Interoperability Profiles for Building Automation: Ron Bernstein, RBCG Consulting

Test Program Development: Ravi Subramaniam, IEEE Conformity Assessment Program

Asset Deployment: Alvin Razon, National Rural Electric Cooperative Association

Howard Self, ABB System Verification Center

Figure 2.2. Panel Participants 


\subsubsection{Panelist Highlights}

Interoperability Profiles for Building Automation: Ron Bernstein, RBCG Consulting.

A comprehensive design and specification program includes products, people, and systems as shown in Figure 2.3. Product T\&C can cover the physical interface, logical interface, and functional interface (three attributes of a product). The logical interface includes communications, and this is the major focus for interoperability. Physical attributes are also important, including the device profile; inputs and outputs; and sensors, actuators, and controls algorithms. One needs to know how to control, monitor, and the system, and its sensors and actuators. Contextual information alongside sensor measurements provides a full picture of the implications of data. A well-defined communications interface requires a common model (e.g., 7-Layer Open System Interconnection (OSI) Model), which feeds into the product profile definition (controls, network variables, etc.), and leads to a welldocumented specification.

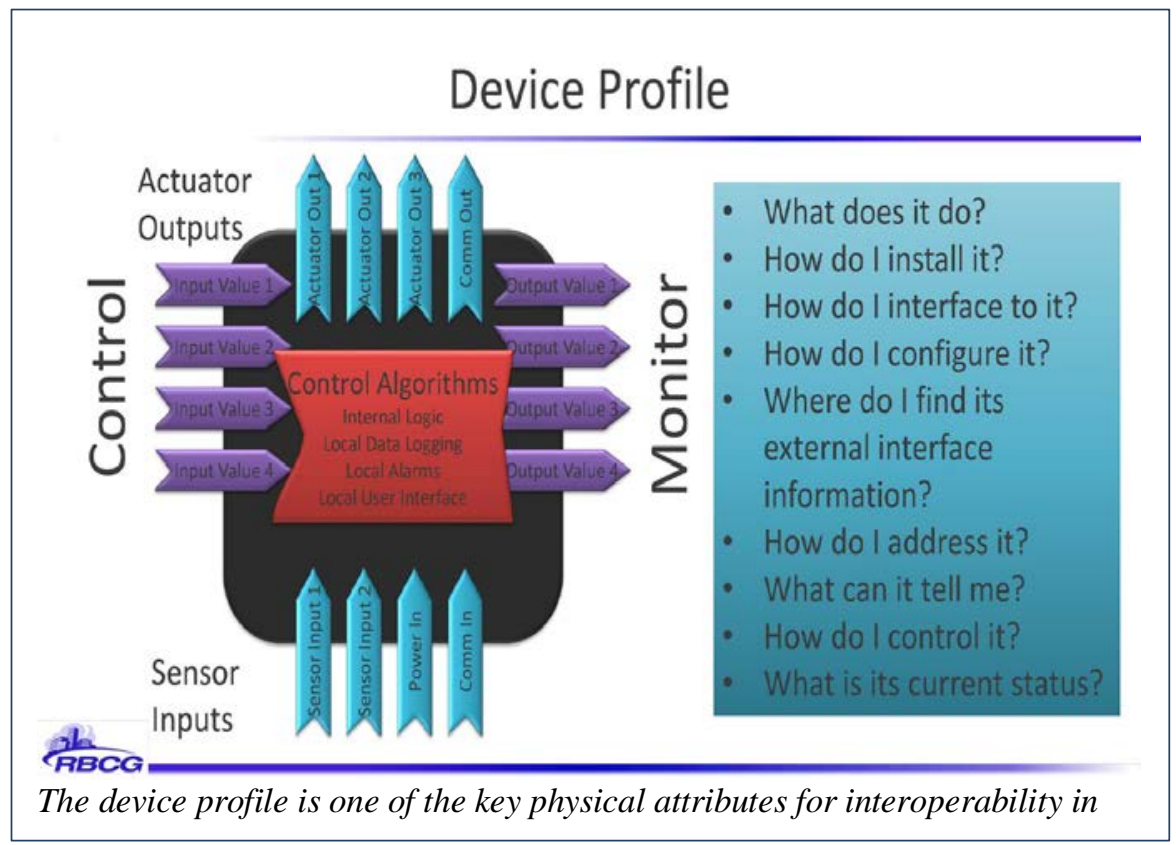

Figure 2.3. Elements of a Device Profile

There is a need to test and certify people. There is a need to develop training programs for product designers, system designers, and others to provide verifiable proficiency and professional credentials. Subject matter experts (SMEs) with requisite skills and abilities need to be involved to build core capabilities. They can also share advice on prior successes with testing and certification.

Interoperability is driven by who in the system has the problem but requires an upfront cost for development by the equipment vendor. We need to develop a process around conformance for interoperability, and well-defined product interfaces. An example is system testing in buildings. How do we define the interactions between the various products in the building? Well-defined system integration requirements are required for testing and 
certifying interoperability of the entire building (e.g., for HVAC, lighting, safety, gas, utilities, security, energy, fire, and network communication). Testing and certifying enterprise systems requires a commissioning plan and certification process for groups of buildings.

Test Program Development: Ravi Subramaniam, IEEE Conformity Assessment Program (ICAP). The IEEE provides a platform for their constituents to develop solutions and standards, with an emphasis on interoperability. Implementation of testing requirements occurs within standards; T\&C programs form within IEEE when there is impetus from a stakeholder community.

ICAP is part of standards implementation within the IEEE standard's lifecycle that is depicted in Figure 2.4. ICAP has roughly 1,000 active standards with 500 under development, including a few of programs focused in this area. There are a number of challenges to standing up a testing, certification, and interoperability program. There must be a demand, i.e., a value proposition that drives the need for T\&C. Outside expertise is also required to develop T\&C programs, as it does not exist within standards working groups. T\&C programs require dedicated time and resources so the value proposition must be clear.

\section{The IEEE Standard's Lifecycle}

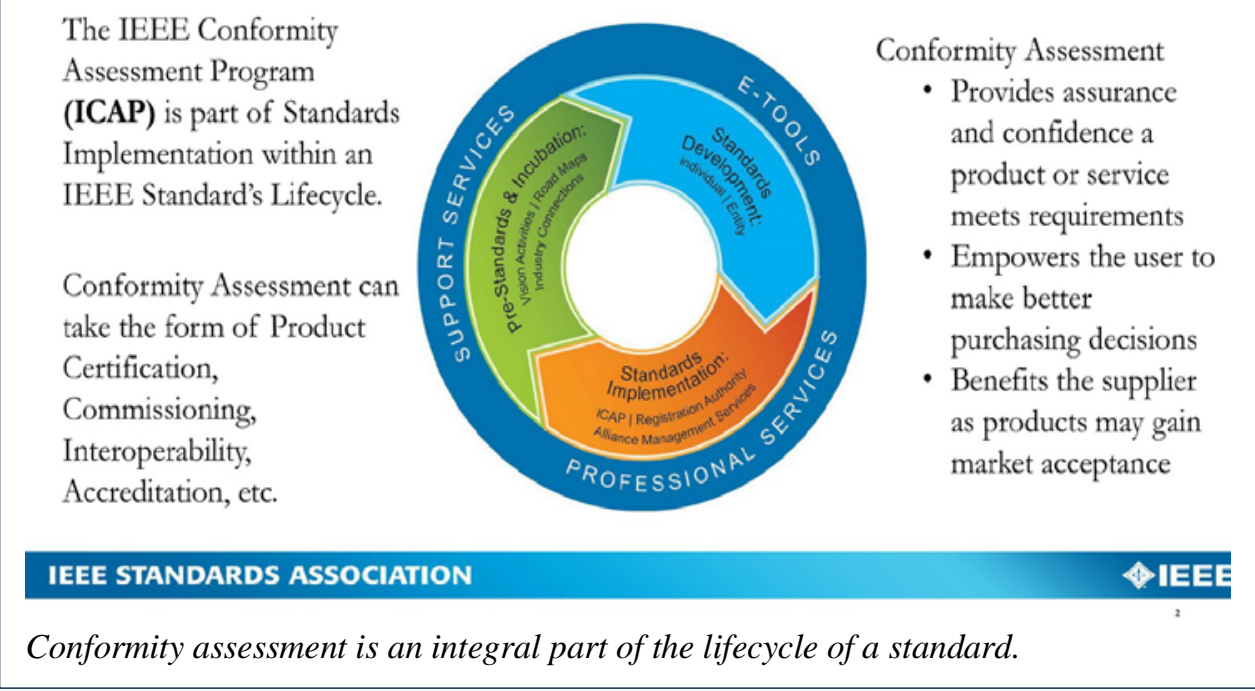

\section{Figure 2.4. The IEEE Standard's Lifecycle}

When there are market drivers, the end-users want T\&C, and it makes sense to develop a program. History demonstrates the importance of certification. An example is the deployment of synchrophasor systems under the American Recovery and Reinvestment Act of 2009 (ARRA), which made a significant contribution to the market [4]. NIST later performed an investigative study [5] of deployed phasor measurement units (PMUs) and found eight out of 10 tested were not compliant with the IEEE Synchrophasor Measurement TSS (test suite specification). 
Asset Deployment: Alvin Razon, National Rural Electric Cooperative Association (NRECA). In the past, expensive and unsecured custom interfaces were the norm. About \$3 billion of Department of Energy (DOE) ARRA grants went toward research on custom interfaces, which are not only expensive but have more potential attack vectors. Today there are new solutions. The NRECA Distribution Optimization (DO) Team is developing the Multi-Speak ecosystem of practical solutions shown in Figure 2.5 to "enable the value of data" for safety, distribution reliability, and integration cost reduction. Over 800 utilities in 21 countries use Multi-Speak; the objectives of Multi-Speak are to provide a cost-effective, real-time, cyber-secure, scalable, testable and certifiable solution. It supports over 40 systems to enable interoperable communications, helping to minimize the risk of technology integration. Multi-speak has enabled an advanced metering infrastructure (AMI) to Outage Management System (OMS) for WIN Energy, saving over \$60,000/year. In the future, the objective is to have interconnected systems enabling real-time 2-way data and energy, reaping benefits in the form of lower costs for capital equipment, outages and restoration, cybersecurity, and resilience.

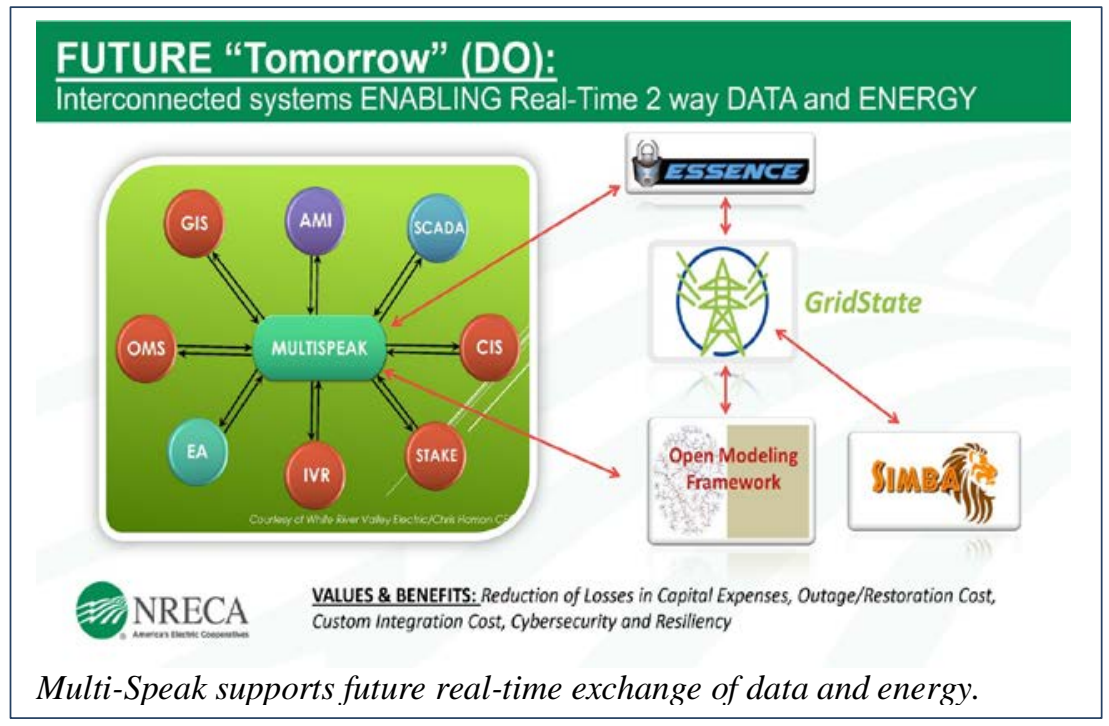

Figure 2.5. Multi-Speak Ecosystem

NRECA currently provides online Multi-Speak training for engineers and CEOs; an online testing harness that tests function sets instead of individual functions; and supports a marketplace for selling and buying Multi-Speak compatible applications. Use cases documenting actual cost savings reported by utilities are also available on the Multi-Speak website (https://www.multispeak.org/).

Manufacturer Perspectives: Howard Self, ABB System Verification Center. ABB maintains a lab for conducting system conformance testing. From ABB's standpoint, interoperability is really about usability for equipment. If equipment always performed as expected, $\mathrm{ABB}$ would not require an expensive testing lab; each of the $\mathrm{ABB}$ business units sponsors 10 full time employees (FTE) per year to conduct testing at a cost of $\$ 1$ billion 
annually. Interoperability is so poorly assured that ABB even has to re-test the conformance tests before they start integration and address interoperability.

After its introduction, ABB implemented the IEC 61850 standard in its product portfolio and established the ABB System Verification Center to verify correct implementation. The IEC 61850 series provides interoperability between intelligent electronic devices (IEDs) from different suppliers or between systems for power utility automation that reside in equipment/devices from different suppliers. Manufacturers have different capabilities, so testing of some features may be optional, depending on the manufacturer. The optional tests may be critical to interoperability, leading to some of the challenges we are facing today. In this test center, ABB tests every product, system component, application and tool to demonstrate functionality and performance. The rationale for testing is summarized in Figure 2.6.

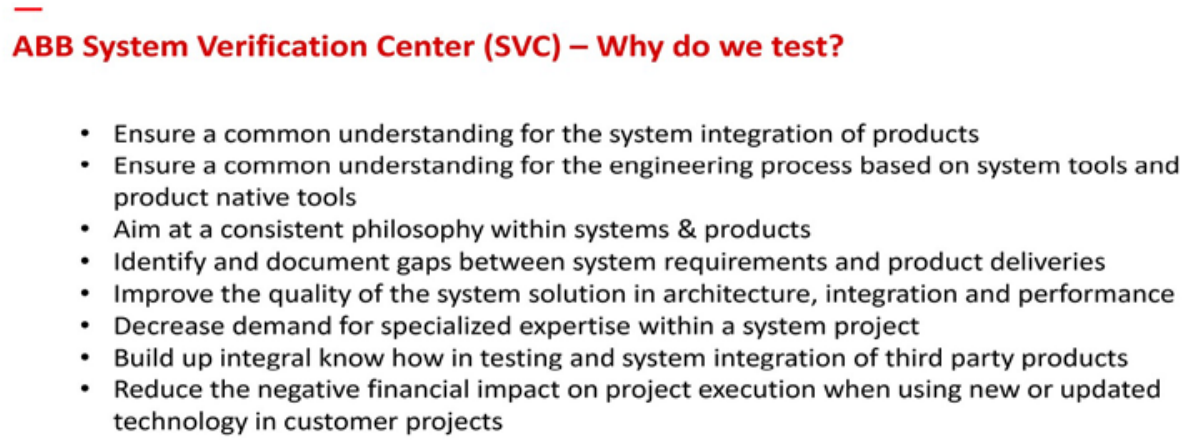

Figure 2.6. ABB's Rationale for Testing

Testing is important to approve components and systems so that they operate appropriately. Interoperability really comes down to usability testing. The types of test that should be run include the Conformance Type Test (stand-alone product test); Product System Test (single product verification in a small, stable system); and System Verification and Validation (system integration test in a reasonably large system). Device hygiene, which includes managing firmware updates, can have a significant impact on interoperability and associated T\&C requirements.

\subsubsection{Summary of Panel Q\&A}

Software and firmware interoperability: Most of the discussions focused on equipment. Many projects today focus on software to upgrade to new systems (e.g., AMI systems). For example, the utility has software from a vendor, but wants to upgrade to a different package. The software upgrade will need to be coordinated with a corresponding firmware update for the affected devices to ensure they are still interoperable after the upgrade. 
T\&C needs to focus more on software packages and systems outside the substation. The same model, framework and challenges apply to both equipment and software. Interfaces and document requirements must be fully understood to write specifications. Vendors will not develop solutions and testing unless specific requirements are defined. A question was raised on how to improve interoperability for AMI standards. There are standards for AMI, but the system is not interoperable because of the many options allowed by the standards, such as multiple data tables. At this time, the head-end system from one vendor will not work with a competitor's meters because of vendor specific implementations of the standards options. This issue showcases how a system does not have interoperability even when it is covered by a set of standards.

A few years ago, the Association of Edison Illuminating Companies (AEIC) attempted to bring together East Coast utilities to develop a common set of data tables for smart meters in an effort to enable interoperability between meters and head-end systems from multiple vendors. The development work resulted in five common data tables for meters. However, the end results were not formalized into requirements for adoption because the AEIC membership did not approve the outcomes.

Drivers for $\mathbf{T} \& \mathbf{C}$ in interoperability standards: The objective is to achieve systems and components that communicate with each other. For ABB, testing is an important aspect for the company and its customers. The goal is to understand what to test - is it everything, or a subset based on what the testing committee agrees to? The objective is testing that is meaningful. Even when standards are developed, end-users do not always include them in specifications or RFPs or require customers to buy standardized products. Note that close engagement with customers on testing and resolving issues likely enables equipment vendors to achieve product certification.

\section{Smart Grid Interoperability T\&C Challenges}

Discussions revolved around a series of focus questions about challenges for T\&C programs (Figure 3.1). The objective was to understand some of the key challenges that are inhibiting the development and application of effective T\&C programs that pertain to the interoperability of smart grid systems and components. Discussions included technical (technological, standardization, measurement, etc.) as well as non-technical (economic, regulatory) challenges and considerations. The sections below summarize the challenges identified in both areas. 


\section{Focus Questions for Challenges}

Why is T\&C not currently working for interoperability at the needed levels?

- What are the major issues and technical challenges to overcome to catalyze the development of T\&C programs?

- What are the major challenges impeding implementation of T\&C programs?

- What are the business/economic and other challenges that limit T\&C implementation and program development (e.g., market, institutional, regulatory, etc.)?

Why are T\&C components not integrated more within interoperability standards?

Why do so few T\&C programs exist for smart grid standards?

\section{Figure 3.1. Focus Questions for Challenges}

\subsection{Technical Challenges}

A number of themes emerged as major technical challenges, described below. Table 3.1 provides the set of complete responses in each area.

Variation in certified functionalities: Inconsistencies in how the requirements of standards apply, to both functionality and technologies, can inhibit T\&C or make it more difficult to apply.

Data quality, availability, and transparency: Without good quality data, T\&C can be inaccurate and incomplete; timeliness and sufficiency of measurements play a role.

Harmonization of standards and protocols: Interoperability standards span many different devices, technologies, protocols, and domains; mapping these many standards to work in harmony and defining minimum requirements is challenging.

Technology and infrastructure: Technologies and capabilities continue to evolve, outpacing standards development; multi-level devices and related interoperability requirements pose greater challenges for T\&C. Bridging protocols for Cyber-Physical Systems (CPS), the IoT (Internet of Things) and IIoT (Industrial Internet of Things) to a common framework is a challenge.

Current standards landscape: The standards landscape is vast and difficult to navigate; some standards are outdated, non-testable in their current forms, too specific, overlap, or inconsistent with international compliance. Standards are continuously evolving, making it difficult to keep up with requirements and to formulate long-lasting, relevant T\&C programs. International harmonization effort will be useful. 


\title{
Table 3.1 Technical Challenges for SG Interoperability T\&C Programs
}

\author{
Variation in certified functionalities
}

- Addressing variations in optional functionality in standards, where users can select different 'options' or functions (but not all) and still meet the standard. Similar products can be certified to a standard but not meet the same requirements for functionality.

- Lack of capability for line item certification; an example is IEC 61850. The system or product can be compliant in different options or lines, but does not need compliance with all parts of the standard to gain certification.

- To test and certify, the product has to respond to specific functionalities (e.g., IEEE 1547-2018 requires advanced functionalities).

- Gaining agreement on what is being tested and certified; clarity on what functions are most important for conformity; and what constitutes a passing grade.

- Non-harmonization of requirements; for example, the same DER System may have different T\&C requirements for independent system operator (ISO) and distribution electrical power systems.

\section{Data quality, availability, and transparency}

- Complexity and lack of data; without good data, T\&C can give incomplete and inaccurate answers; real data is essential. A solution is to start accumulating data (although utilities do not always want to share data), and be able to scale. Utilities need to analyze the data to see if it contains situations not covered by the standard. Definition of what is good data needs to be consistent between the standard's requirements and the data curation process of the utility.

- Obtaining sufficient measurements

- Timeliness of the data,

- Time synchronization of inputs and accurate time-stamping of measurement samples and events for impact analysis.

- Applications depending less on physical abilities and more on communication interfaces between them.

- Market testing is now more a function of communications and interoperability, driving need for T\&C program around non-physical aspects.

- Companies do not like to share real data and may manipulate data if it applies to billing.

Harmonization of standards and protocols

- Smart grid standards harmonization and integration, i.e., mapping standards to work together in harmony, and taking the best of both IEC and IEEE standards. Example: IEEE DNP3 and IEC 61850 mapping.

- Creating a "universal” performance metric separate from technology, function, or standards.

- Lack of a minimum set of agreed upon features to build certification programs.

- Challenges in creating protocol bridges among the Internet of Things (IoT), Industrial Internet of Things (IIoT) and Cyber-Physical Systems (CPS) to a common framework. Standards groups could potentially explore the concept of 'software test rigs' to address this.

- Ability to harmonize IoT and new technology/semantics; it is not possible to bridge all protocols, domains, and applications (e.g., bridging to unique Department of Transportation Standards). Useful solutions could be development of a common semantic model, building into mobile apps, and integration with secure data sharing. A NIST provided taxonomy - OWL (Web Ontology Language) or more - could also be helpful. 
- Ongoing evolution of smart grid power systems and dynamic technology landscape; the capabilities of systems are outpacing standards and emerging applications, which is nearly always true in the world of standards; standards are often a response to market-based technology drivers.

- Complexity of interoperability; integrating multiple systems used by different suppliers; T\&C for systems is more difficult than for individual products.

- Some technologies are more difficult to certify; existing methods for interconnection may not work with emerging technologies, creating inefficiencies in the T\&C process if it cannot keep pace with innovation; more difficult for utilities to certify based on evolving technologies.

- Providing T\&C for multi-level interoperability and interfaces in devices and systems.

- Lack of uniform hardware specifications affects information model for microgrid controllers.

\section{Current standards landscape}

- Multitude of standards requires harmonization efforts to solve current interoperability issues; dealing with many updated versions that get published; understanding which standards matter and why, and which are not working; too many standards for labs to test to (creates an unsustainable business model). One solution is to curate standards according to the number of products using the standard, or the utilities buying products.

- Lack of requirements for smart grid standards by end users; identifying smart grid standards that are most suitable for T\&C.

- Not all standards are testable as written or in their evolved form; these may require test plans. T\&C programs can prioritize standards for testing.

- Different standards for testing among various bodies around the world; global compliance and overlapping international requirements increase the complexity of the standards landscape.

- Special solutions for smart grid, specified to smart grid, for communications and functions (e.g., IEEE 2030.5 protocol); some standards are too complex.

\subsection{Non-Technical Challenges}

Non-technical challenges for interoperability T\&C programs revolve around several key themes relating primarily to economics and markets, as described below. Table 3.2 provides the set of complete responses in each area.

Value proposition and business case: The cost-benefits associated with interoperability and conformance are not well-understood, so it is difficult to justify the expense of testing. Insufficient value proposition and business case for smart grid interoperability creates challenges for developing T\&C programs as well as establishing certification labs.

Market and technology uptake: Slow adoption of smart grid technologies and lack of end user demand limit the market drivers for T\&C programs. Investment in such programs is slow and limited; other investments such as those driven by safety or Public Utility Commission (PUC) requirements take precedence.

Institutional awareness and commitment: Utilities have limited involvement with standards development and related T\&C programs; RFPs often do not require $\mathrm{T} \& \mathrm{C}$, and clarity on need for $\mathrm{T} \& \mathrm{C}$ is lacking. 


\section{Table 3.2 Non-Technical Challenges for SG Interoperability T\&C Programs}

\section{Value proposition and business case}

- Considerations in adopting T\&C programs include tradeoffs between intellectual property and scalability, strategic advantages for conducting T\&C, and funding.

- Insufficiently developed value proposition and business case for interoperability T\&C and certification labs in the smart grid industry. Industry requires development of an economic model and justification for when T\&C gives a return on investment.

- Reaching economies of scale with standards and certification activities. Various T\&C bodies operating under a single umbrella would reduce the marginal costs associated with T\&C.

- Limited availability of data to support interoperability cost and benefit analysis.

- Lack of clearly defined cost vs. risk analysis to support certification; conflict between program startup cost versus life cycle cost can hurt organizations over the long term if they focus on one over the other. Utilities often follow lowest cost bid awards, not Total Cost of Ownership; procurement requirements typically do not specify only tested/certified products on bids, therefore little motivation exists for manufacturers to certify. Short cycles of technological change also influence the cost structure for business and industries.

- Cost of T\&C - de-risking technology integration to lower the cost of integration (i.e., who will pay for the development of T\&C).

- No urgency in supporting interoperability T\&C programs compared to other priority areas such as safety or cybersecurity.

\section{Market and technology uptake}

- Insufficient market pulls for T\&C for interoperability:

- Relatively slow adoption of advanced smart grid technologies and associated market drivers for T\&C; accelerated adoption would drive urgency for T\&C (i.e., a chicken and egg situation).

- Lack of major end user demand and validation of standards. PUC-driven capital expenditures are not in the same line as new technology adoption.

- Early-stage start-ups trying to create new technology and not worried about meeting interoperability certification or testing - most often they care about product safety certification. Conversely, utilities require decades of support from a company that may not last that long.

- Business decisions will drive the investment in T\&C. Slow and limited long term investment in grid modernization due to uncertainty and the desire to realize maximum network effects. The investment community needs context to invest, i.e., to reconcile operating and capital expense models and different perspectives.

\section{Institutional awareness and commitment}

- Limited participation by utilities in standards development or T\&C. Utilities and vendors do not want to broadcast their limitations, nor do utilities have sufficient resources to send representatives to standards meetings.

- Lack of procurement requirements that target standards and T\&C (e.g., from utilities, service providers); education is needed to help organizations understand the value proposition; vendors have their own test efforts, and end users do not mandate T\&C.

- Engaging utilities to formulate universal requirements; manufacturers are often waiting for aggregated demand to pursue standardization/compliance. Over 3,000 utilities in the United States. An Open Distributed System Platform (DSP) could get major utilities to agree on system requirements to help move toward addressing DER challenges. Utilities can determine what other standards are needed and move toward a T\&C framework with available standards.

- Limited understanding of the effects (and benefits) of T\&C on users - individuals, businesses, and vendors; pathways for educating end users (utilities) via more end user data and better understanding about devices; educating communities of interest on the business case for T\&C.

- Workforce issues (expertise in T\&C) compounded by the complexity of testing; testing experts are rare and expensive - expertise needs to be more distributed. Workforce can test to certain standards without a lot of SME knowledge, with training. Issues arise in understanding what items are testable and how to go about testing. Test professionals can write test cases but need in-house clarity behind the testing requirements. 
- Communicating costs of "interoperability" to different decision makers; need to develop a general Q\&A primer with examples for different stakeholder groups (regulators, executives). Use analogies such as the Massachusetts "Fast Lane" highway toll transponder versus New York and New Jersey "EZpass" which forced use of two transponders.

- Lack of clarity on T\&C requirements; limited perspective of T\&C as a later step after there are only a few 'competing' standards.

- Apparent resistance among commodity providers; need to level the playing fields to enable large and small organizations to contribute.

\section{Regulatory}

- Potential economic barriers to conformance created by the overarching regulatory structure in the U.S.; lack of uniform legal approaches in the U.S.; and lack of federally mandated standards as on the electrical side.

- Uniform interoperability that makes sense through the entire national grid, not just state jurisdictions; current PUC model defeats the purpose of having an interoperable national grid. The transformation of the energy sector is increasingly blurring the jurisdictional line between the Federal Energy Regulatory Commission (FERC) and PUC, creating a need for greater FERC engagement.

- Articulating to regulators what direct and indirect value T\&C provides (e.g., potential use as cyber/physical safety oversight tool, nondiscriminatory grid access transparency tool - makes it more difficult for the utility to manipulate DER interconnection). Ability to provide technical assistance to regulators to underscore the importance of standardization is limited.

- Lack of technical understanding among regulators; need to inform and educate (smart grid is heavily regulated).

- Lack of clear business case for T\&C to put before regulators.

- Poor understanding by regulators of value proposition for T\&C (or standards), a situation much like that for cyber security in the past.

- Navigating regulatory silos and rules (i.e., home-based vs. utility data).

\section{Smart Grid Interoperability Profile Elements}

Discussions revolved around a series of focus questions about interoperability profile elements (Figure 4.1). The objective is to identify some of the key features to specify for three major elements where interoperability comes into play: 1) physical assets/devices, 2) communication protocols, and 3) information models.

\section{Focus Questions for Interoperability Profiles}

Physical assets -What are the aspects of the physical performance specifications that contribute to interoperability?

- What aspects of the physical asset description are required or relate to interoperability?

- What features of the physical asset do we need to specify? On the utility or customer side of the meter?

- Does it matter where you place the physical asset or device in the system, or the service it is providing?

Communication protocols - What aspects or features of the communications protocol will be useful to profile?

Information models - If domains are moving toward simulations, what is the subset of data/model fields that will be most useful and valuable to specify for a profile?

Figure 4.1 Focus Questions for Interoperability Profiles 


\subsection{Profile Elements for Physical Assets}

A number of themes emerged for interoperability profiles for physical assets, described below. Table 4.1 provides the set of complete responses for this area.

Physical assets. Physical connections and interfaces of devices and systems are important points of interoperability in physical assets. Plug-ins to other systems (e.g., storage) and devices play a key role. Function, performance, and connectivity are some of the key asset categories to profile. Common specifications for physical lockouts are important, for example. End nodes need to be responsive to a control hierarchy; improved simplicity would help the uniformity of controls strategies.

Compatibility of standards allows for efficient and effective communication and requirements specification for when/how systems and devices work together. Specifications need to cover how devices interact; the profile should focus on features that allow devices/systems to coordinate. Two different sensors using older communications protocols can provide a needed level of interoperability, but may not necessarily maximize the performance/capabilities of a specific hardware platform.

Energy transfer and power conversion equipment need specific requirements and specifications for each to be able to maximize performance. Equipment/assets need to operate appropriately and then be able to communicate with other systems; this requires specification of interface behaviors.

Asset functions: Asset functions are physical but also relate to cybersecurity, performance, and operations. They provide semantic context for the information exchange. This raises the question of whether interoperability depends on the application. If each application needs an interoperability profile, how can different types of profiles be delineated. The physical context of operation and environmental factors will help determine the appropriate approach. Important elements to profile include many of those related to reliability and security, including resistance to physical/ environmental events (e.g., lightning), protection from physical, cyber or other attacks, and performance. In addition to cyber interoperability, physical interoperability and compatibility are also factors to consider. 
Table 4.1 Smart Grid Interoperability Profile Elements for Physical Assets

\begin{tabular}{|l|l|}
\hline Physical assets & Asset functions \\
\hline - Physical connections/interface & - Physical \\
- Shape plug and play (installation is expensive) & - Self-declaration of capabilities \\
- Electrical and data connections, including RF & - Electrical and communications lock-out, tag-out \\
spectrum and modulation limits to SDR (e.g., & and fail-safe/operational \\
electric vehicle (EV) charger, how long can it & - Lightening resistance \\
provide data) & - Specification of device behavior(s) for "physical \\
- Unique physical device interconnections & interoperability" \\
- USB 2.0 port & - Broadened context of physical asset \\
- Reliability of sensors/transducers, contingency & - Environment \\
requirements & - Functions \\
- Physical standards & - Resistance to attack by radio frequency (RF) \\
- Switches, connectors and data connection cables & interference, cyber threats, or by physical \\
(vendor-specified), meter connection, etc. & malfeasance \\
- Plug-ins to other systems and devices & - Identification of existing device physical asset \\
- Data storage (cloud computing) & performance specifications \\
- Timing (information within a period of time) & - Identification of functions most relevant to operation \\
- Speed & and inter-operation \\
\hline
\end{tabular}

\subsection{Profile Elements for Communication Protocols}

A number of themes emerged for interoperability profiles for physical assets, described below. Table 4.2 provides the complete responses for this area.

Information exchange characteristics: Information characteristics that are important to profile for interoperability include the type of information transmitted and the nature of the device, the characteristics of that information, and the communication protocol and/or mode. Language, ontology, and terminology of information exchange are key elements to cover in a protocol to support interoperability.

Communication profile elements: As shown in Table 4.2, performance or security are primary considerations for communication protocols. Data format, characteristics, timing, speed, and transport are key performance parameters. When considering large volumes of data transfer, protocols also need profile equivalents for the subset of information needed to communicate to enable interoperability. When translating/encoding at the subset level, information may be lost and should be addressed or captured.

A common protocol implementation conformance statement (PICS) specifies what it means to comply with a protocol (e.g., IEEE 802.XX). This is the basis of interoperability and the core of an interoperability profile for communications. Protocols should preserve the semantic models but need to be extended (add new elements) as necessary. International requirements and issues also need to be considered. 
Table 4.2. Smart Grid Interoperability Profile Elements for Communication Protocols

\begin{tabular}{|c|c|}
\hline Information exchange modes & Communication protocol elements \\
\hline $\begin{array}{l}\text { - Type of information the device can communicate } \\
\text { and characteristics } \\
\text { - Protocol specification over multitude of } \\
\text { communication media, e.g., for radio } \\
\text { frequency (RF), cellular, mobile host (MH2), } \\
\text { programable logic controllers (PLCs), etc. } \\
\text { - Mode of communication (data movement } \\
\text { mode, i.e., simplex, half-duplex, full-duplex, } \\
\text { etc.) } \\
\text { - Internet Protocol } \\
\text { - Well-developed virtual models of equipment and } \\
\text { systems. } \\
\text { - Profile language guidelines for different levels of } \\
\text { users and developers. }\end{array}$ & 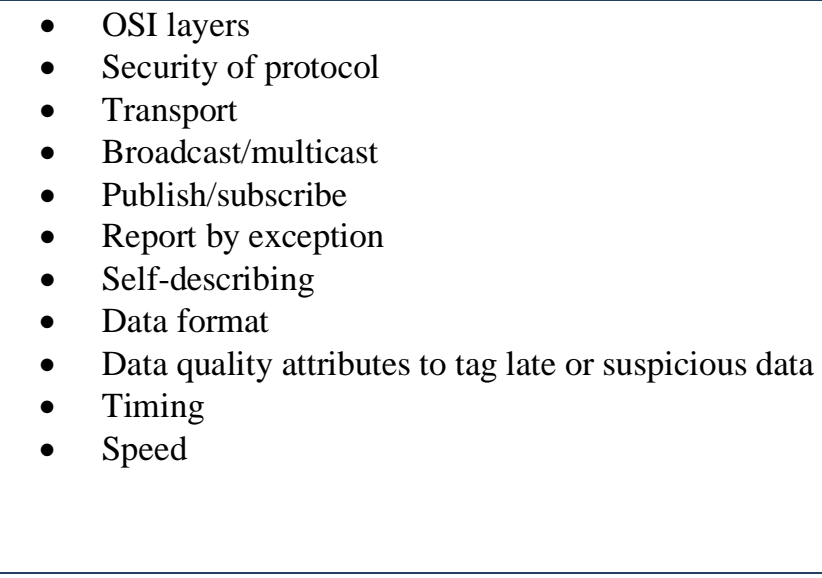 \\
\hline
\end{tabular}

\subsection{Profile Elements for Information Models}

A number of themes emerged for information models, described below and in Table 4.3. The performance and utility of information exchange, analysis and interpretation are key themes.

Uniform software units and data fields, as well as data accuracy, speed and resolution are important profile elements. Response fields, query format, and response format for information models are also required. Without specifying these fields, the information is meaningless; it must interact with the rest of the system as needed. Degrees of freedom can be constrained to specify what the users and developers want to work with.

It is also easier to standardize profiles for data subsets instead of taking a top down (i.e., higher level) approach. In the absence of profiles, it could be possible to organize information at a high level and then extract or dissect it as needed.

In practice, each device currently has a subset of mandatory features for communicating with other devices, but that does not necessarily enable or improve interoperability. A core set of standards (e.g., CIM, IEC 61850, Facility Smart Grid Information Model [FSGIM]) can serve as a backbone to map to other standards, then common elements identified.

One question raised is whether it is possible to put "bounds" on behaviors, i.e., the ranges of parameters that should be specified for operation, or device behavior. This adds a semantic dimension to the information mode. Bounds would depend on the complexity of the information model and the devices included in the system.

Smart city ecosystems are a good example. Devices have to be able to communicate within different environments and across domains. Each domain (e.g., city planning, emergency response, traffic, etc.) has its own set of protocols and solutions for information models, making interoperability more challenging. 
Table 4.3. Smart Grid Interoperability Profile Elements for Information Models

- Data

- Speed and accuracy in sending/receiving (Information Read Response Time)

- Storage

- Data resolution of sensors; resolution with the same percentage

- Refined relational database (i.e., common databases)

- Software and simulations

- Uniform software (units, data fields, and range of values)

- Required model fields, query format and response format

- Decomposition of information models into a set of function blocks

- Testing of software and models

- Vendor configurable for an application

- Applicable in smart cities and many other sectors or domains

- Networks and infrastructure

- Discovery and self-documentation (external interface file); this feedback to plug \& play

- Flexible; remote firmware amenable to network discovery

- Time aspect protocol and Internet Protocol (IP) address; time aspect refers in part to time synchronization between related data points

- IP address equal to 'blank' (for security); ability to accommodate media access control (MAC) addresses

\section{Approaches for Developing T\&C Programs for Interoperability}

As noted previously, while current T\&C programs for interoperability exist today, they are insufficient to address the rapidly accelerating use of new devices and systems connected to the grid. T\&C for interoperability could help to facilitate grid modernization by assuring confidence in the way new systems connect and perform.

Discussions revolved around a series of focus questions on approaches for designing $\mathrm{T} \& \mathrm{C}$ programs and encouraging acceptance and buy-in (Figure 5.1).

\section{Focus Questions for T\&C Program Approaches}

- What will encourage/enable development/buy-in for an interoperability T\&C program?

- Should we incorporate T\&C into regulation or procurement language?

- Are market conditions right for T\&C programs?

- What are some of the key elements for designing successful T\&C programs?

\section{Figure 5.1. Focus Questions for T\&C Program Approaches}

\subsection{Potential Pathways to Buy-in and Acceptance of Interoperability T\&C}

There are a number of factors that could influence more widespread use and acceptance of $\mathrm{T} \& \mathrm{C}$ for interoperability. If regulators require T\&C in utility RFPs and procurement, then the market will respond accordingly; this could be a sound approach/strategy to drive change. 
For the many different actors interacting on the grid, IO T\&C could serve as a tool to meet needs for managing diverse energy resources. T\&C programs can also provide a clearinghouse for data provided by various stakeholders - creating more value for constituents. A potential path to gain buy-in for interoperability T\&C programs could follow several stages, outlined below.

- Mandate- Regulation can serve as demand driver for T\&C programs. Regulators can take further action on behalf of all stakeholders to drive progress. The PUC and/or the Public Service Commission (PSC) adopt and mandate the suite of T\&C requirements. Standards covered by the T\&C program may have safety critical implications as the energy sector moves toward grid modernization. There is a need for utility associations (e.g., EEI, APPA, NRECA) to educate how standards and testing are being integrated into critical energy sector operations. It is important to consider the needs of legacy utilities during a transitional decade to minimize disruption - whether business or grid operations. Different approaches and needs for T\&C will depend on where utilities are located. Specialized industry organizations, such as the Electric Power Research Institute (EPRI), can provide means to translate and mandate requirements to appropriate entities.

- Definition - Utility operators should require interoperability profiles and standards (through procurement language or otherwise) to maximize benefits to the system. Procurement serves as strong driver for T\&C. However, procurement specifications need to define specifications with real long-term economic value for suppliers to comply with T\&C. These could include performance metrics, best practices, guidelines and specifications, drawn from best practices. T\&C programs take the responsibility away from vendors and help make the landscape more successful. They provide a goal for vendors to work toward regarding interoperability. Smart Contracts could address certain conditions by utilities as well as concerns of owners; capabilities could be certified and tested. Some questions to answer include the role of Block Chain (if any), and what happens if a device does not cooperate or comply.

- Testability - T\&C can help validate performance and keep the market fair, where the definition of 'fair' depends on the entity defining it. T\&C programs should not have conflicting requirements.

The business case for T\&C needs strengthening to encourage wider support. There is a government role to help strengthen the business case for testing labs, e.g., via Federal funding for open source testing services, and other enabling approaches or technologies that help test labs to enter the market and develop testing approaches. If test labs, vendors and users can gain access to test harnesses, then more user groups can join the early adopter pool. One approach is to view the utility as a 'customer' for T\&C, recognizing that different business models exist for utilities and that each will react accordingly. Benefits should be clear to these various implementers of T\&C programs (Figure 5.2), such as lower service costs to customers. The product development emphasis should be on competitive advantages while the objective of standards should be on baseline performance thresholds and interfaces only, to avoid constraining innovation. 
Business Models of Utility T\&C Programs

- Investor Owned

- Federal Power

Utilities (IOU)

- Cooperative

- State Chartered

- Municipal

- State-Owned/ IOUManaged Hybrid

\section{Figure 5.2. Business Models of Utility T\&C Programs}

\subsection{Design Approaches for Interoperability $T \& C$}

Possible program designs for T\&C (Table 5.1) center around developing reasonable, consistent testing programs for minimum levels of interoperability, backed up by reference sets and use cases, and specifications for performance (e.g., speed, accuracy, etc.). Programs that are robust, transparent and have multiple deliverables will be more user friendly, improve confidence, and mitigate risk. Education on the value (e.g., cost, performance, life cycle) of utilizing $\mathrm{T} \& \mathrm{C}$ is a key requirement in all approaches.

\section{Table 5.1. Smart Grid Interoperability T\&C Approaches}

\section{General testing strategies}

- Determine minimum subset of existing protocols for T\&C

- Develop common test bed to avoid state-by-state solutions and regulations/rules

- Learn from tests of Plug-fest; devise new tests

- Conduct test for integration, with focus on interoperability

- Set rules for speed, accuracy, etc.; develop use cases, then test and adjust

- Determine device level inputs/outputs (start with the information model, determine the suite of devices, and identify points of communication)

- Create a defined profile to level market baseline requirements, backed up with use cases and reference sets

- Set aspirational T\&C targets: Distributed Energy Resources (DERs), Commissioning Conformity T\&C

\section{Reference sets and use case resources}

- Utilize the ANSI website with 30 use cases as a starting place for reference sets

- Access standard RFP language vetted by many, for microgrids, vehicles, and other cases

Program constructs

- Develop robust and transparent T\&C programs with multiple deliverables, including:

- Clear and understandable procedures

- Use case examples

- Education and awareness

- $\quad$ Robust and transparent testing requirements

- Develop suggested procurement language for RFPs; create corollary explanatory document for PSCs/PUCs

\section{Smart Grid Interoperability Scenarios Priority Interfaces}

\subsection{Conceptual Models for the Smart Grid}

Four conceptual communication scenarios are under development as part of NIST's activities to update the Smart Grid Interoperability Framework 4.0. ${ }^{1}$ The electrical grid is undergoing a

\footnotetext{
${ }^{1}$ Workshop Plenary Presentation: Avi Gopstein, NIST. Link to presentations found in Appendix C.
} 
transformation, and new pathways of communication are emerging. The increasing integration of Distributed Energy Resources (DERs), technology diversity, and emerging functional overlaps across the transmission, distribution, and customer domains are creating challenges for communications interoperability. An intelligent distribution system is emerging, with improved controllability and intelligence. Devices are now behind the meter to an extent never before seen, introducing complexity and challenges that are difficult to manage and anticipate. Conceptual diagrams developed to describe different scenarios for communications pathways in the modern or future grid include the following:

- Legacy Communication Pathways Scenario - based on previous models of communications and interoperability profiles and frameworks.

- High DER Communication Pathways Scenario - includes overlap of Generation Including DER domain with Customer domain. Includes assets on the grid that may be for services other than generation but still delivering electrons into the system. In this scenario, grid devices are likely to have a growing compatibility with Internet Protocol (IP) based communications.

- Microgrid Communication Pathways Scenario - microgrid controller is the point of common coupling (PCC), but who operates the microgrid-utilities, customers, or others-may affect the relevant communications protocols and the type of interoperability they are pursuing.

- Hybrid Utility Communication Pathways Scenario - the "edges of the grid" are system and domain specific, as are the communications pathways interfaces and associated drivers for interoperability requirements. Because the grid and infrastructure and how they are used is evolving, in this scenario even legacy communications may need a mix of protocols to consider for device integration at the edge of each domain.

\subsection{Priority Interfaces for Interoperability}

Discussions covered a series of focus questions about priority interfaces within communications scenarios for different grid architectures (Figure 6.1). The overarching objective is to understand the priorities for ensuring interoperability through T\&C programs, standards, and protocols. Two scenarios were the primary topic of discussions - High DER and Legacy Utility.

\section{Focus Questions for Priority Interfaces}

What critical interfaces should T\&C form around?

- What are the priority interfaces between customer assets within the domain, and between customer or third party assets across domains?

- What are the priority interfaces for inter-domain observability and controllability to/from utility and for utility devices across domains?

Figure 6.1. Focus Questions for Priority Interfaces 


\subsubsection{High DER Scenario}

The priority interfaces identified are primarily at the connection of distributed resources and the grid. Table 6.1 summarizes the complete set of priority interfaces identified. Critical interfaces exist anywhere an operational impact exists.

\section{Table 6.1. Smart Grid Interoperability Priority Interfaces for High DER Architecture Scenario}

\section{$(\leftrightarrow$ indicates priority interface)}

- DER $\leftrightarrow$ Customer; Customer $\leftrightarrow$ Distribution; Distribution $\leftrightarrow$ DER

Most critical interfaces within customer services, hardware installed.

Utility $\leftrightarrow$ DER: Sources owned by utility customers (residential and small commercial) for market-enablement.

- Third Party Providers $\leftrightarrow$ Customer Systems and Distribution Operations

There are specific requirements to enable customer value creation and overall system operation.

- Utility Bus

$\leftrightarrow$ Customer gateway; look at commercial and residential customers to provide them options; need to minimize risk and ensure the quality of the product/service to the customer.

$\leftrightarrow$ Third party bus/gateway

- Substation Controller $\leftrightarrow$ Substation Device

- Distribution $\leftrightarrow$ Customer $\leftrightarrow$ Market $\leftrightarrow$ Operations

This covers a very wide spectrum involving end-users, security and privacy, non-qualified/untrained people, unconventional systems, and bidirectional exchange and power flow.

- Generation, including DER $\leftrightarrow$ Distribution

Issues arise due to different owners, mobility of consumers, and other factors.

- Microgrid Controller

$\leftrightarrow$ System operations

$\leftrightarrow$ Distribution

$\leftrightarrow$ Utility service provider

$\leftrightarrow$ Microgrid participants

Microgrid systems provide customers/end-users a greater influence in the services they receive. Making the end user experience the best possible would help facilitate integration of microgrids and DER into the grid.

- Co-generation/Combined Heat and Power (CHP)

$\leftrightarrow$ System operations

$\leftrightarrow$ Distribution

$\leftrightarrow$ Utility service provider

- Customer $\leftrightarrow$ Distribution/Transmission $\leftrightarrow$ Generation/DER

Customer and third party-owned devices seek to connect to distribution/transmission and provide compensated services. The utility has certain requirements, which may be a barrier to market entry/customer value creation. Interoperability here can lower barriers to entry, enhance customer satisfaction, and provide benefits to the system.

- Building Automation Systems (BAS), Building Management Systems (BMS), Energy Management Systems (EMS) Devices, Equipment $\leftrightarrow$ Grid

These include commercial, industrial, institutional load management and energy management system interfaces; making these systems interactive with the grid would help significantly. Identify interfaces with a welldefined/value proposition - where end users can drive the process, versus the most important or essential interfaces, which require criteria for selecting the priorities. Each device needs to be able to support all IO profiles (harmonization or union of profiles) so manufactured products can be used anywhere.

- Transmission Voltage $\leftrightarrow$ Current Sensing Trial Interfaces

Considering the evolution of this market, one of the most important interfaces is the communication between utilities and third parties. This includes, for example, what 
information they get, how they provide information, and what they need to communicate to the various entities in the framework. There is currently some uncertainty regarding the role of utilities with respect to various stakeholders and end-users in High DER scenarios.

Without T\&C infrastructure, the paradigm for inputs/outputs (IO) among grid entities changes. Large sources of untapped loads and resources need aggregation to scale and achieve the benefit of interoperability profiles. All devices require registration to be able to share authorized information. Data needs to be encrypted to secure the information flows are secured.

The broad variety of customers and distributed resources providing energy to the grid in the High DER scenario poses some challenges. Relationships between the customers and distribution system operators (DSO) are unique. There is currently a lack of knowledge of grid operations on the customer side, and a clearly defined customer interface will be a requirement. In High DER, there are usually different owners, and users want to be able to interact with their data and equipment in an effective way.

There is no specific framework or interoperability profile for managing flexible loads typical of High DER. It is unknown how devices communicate universally, i.e., if devices communicate directly with gateway assets or if devices communicate directly with third party providers or utilities.

\section{References}

[1] Song, E., Nguyen, C. \& Gopstein, A. (2019). Review of Smart Grid Standards for Testing and Certification Landscape Analysis. (NIST-TN-2042). DOI: 10.6028/NIST.TN.2042.

[2] Greer, C., Wollman, D., Prochaska, D., Boynton, P., Mazer, J., Nguyen, C., FitzPatrick, G., Nelson, T., Koepke, G., Hefner Jr., A., Pillitteri, V., Brewer, T., Golmie, N., Su, D., Eustis, A., Holmberg, D., \& Bushby, S. (2014). NIST Framework and Roadmap for Smart Grid Interoperability Standards, Release 3.0. (NIST SP-1108r3), DOI: 10.6028/NIST.SP.1108r3.

[3] OpenFMB Overview. https://openfmb.github.io/\#standard.

[4] Department of Energy (2016). Advancement of Synchrophasor Technology in Projects funded by the American Recovery and Reinvestment Act of 2009. https://www.smartgrid.gov/files/20160320_Synchrophasor_Report.pdf.

[5] Goldstein, A. (2016). 2014 NIST Assessment of Phasor Measurement Unit Performance. (NIST-IR-8106). DOI: 10.6028/NIST.IR.8106. 


\section{Appendix A: Participants}

\begin{tabular}{|c|c|}
\hline Name & Affiliation \\
\hline Vijay Ahuja & Cipher Solutions, Inc. \\
\hline Devesh Ahuja & Cipher Solutions, Inc. \\
\hline Martinez Alfredo & University of California, Riverside \\
\hline Aliya Alimujiang & NRECA \\
\hline Sharon Allan & SEPA \\
\hline Jason Allnutt & IEEE-SA \\
\hline Ron Bernstein & RBCG Consulting \\
\hline Hasnae Bilil & NIST - CPS and SG Program \\
\hline Andrew Bohan & A2LA \\
\hline Paul Boynton & NIST - Engineering Laboratory \\
\hline Rob Burkhardt & PDV Wireless \\
\hline Martin Burns & NIST \\
\hline Steven Bushby & NIST \\
\hline Jorge Camacho & UI \\
\hline Bill Colavecchio & UL LLC \\
\hline Andrew Cotter & SEPA \\
\hline Brian Duffy & McLean Power \\
\hline Nader Farah & ESTA International \\
\hline Avi Gopstein & NIST \\
\hline Jason Handley & Duke Energy \\
\hline Anand Kandaswamy & NIST - Applied Economics Office \\
\hline Jeffrey Katz & IBM Corporation \\
\hline Cach Keyser & A2LA (American Association for Laboratory Accreditation) \\
\hline Ya-Shian Li-Baboud & NIST \\
\hline Rhonda Mackabee & ActioNetInc. \\
\hline Brian Marchionini & NEMA \\
\hline Joel Miller & Merrion Group \\
\hline William Miller & MaCT USA \\
\hline Cuong Nguyen & NIST \\
\hline Cheyney O'Fallon & NIST - Applied Economics Office \\
\hline Thomas Pierpoint & Quanta Technology \\
\hline Alvin Razon & NRECA/MultiSpeak \\
\hline Craig Rodine & ChargePointInc. \\
\hline Angelo Rometo & Cleveland Price Inc. \\
\hline Ruben Salazar & Landis+Gyr \\
\hline Howard Self & ABB Inc. \\
\hline Aaron Smallwood & ESEPA \\
\hline Eugene Song & NIST \\
\hline Ravi Subramaniam & IEEE \\
\hline Chris Villarreal & Plugged In Strategies \\
\hline Randy Wedin & Wedin Communications LLC \\
\hline David Wollman & NIST - Engineering Laboratory \\
\hline Kerry Worthington & NARUC \\
\hline Yanfei Yang & Graphene Waves \\
\hline
\end{tabular}




\section{Appendix B: Agenda}

\begin{tabular}{|c|c|}
\hline \multicolumn{2}{|c|}{$\begin{array}{l}\text { Workshop on Smart Grid Interoperability Testing and Certification } \\
\text { July 9, } 2018 \text { Washington, DC }\end{array}$} \\
\hline 8:00 am & REGISTRATION \\
\hline 9:00 am & $\begin{array}{l}\text { OPENING PLENARY SESSION } \\
\text { - Welcome and Overview of Smart Grid Interoperability Framework 4.0 Avi Gopstein, NIST } \\
\text { - Workshop Overview } \sim \text { Cuong Nguyen, NIST }\end{array}$ \\
\hline $9: 15 \mathrm{am}$ & $\begin{array}{l}\text { KEYNOTE } \\
\text { - Jason Handley, Duke Energy }\end{array}$ \\
\hline $9: 45 \mathrm{am}$ & $\begin{array}{l}\text { PANEL SESSION: VALUE PROPOSITION FOR TESTING \& CERTIFICATION (T\&C) } \\
\text { Moderator: Bill Colavecchio, Underwriters Laboratory } \\
\text { - Interoperability Profiles for Building Automation: Ron Bernstein, RBCG Consulting } \\
\text { - Test Program Development: Ravi Subramaniam, IEEE Conformity Assessment Program } \\
\text { - Asset Deployment: Alvin Razon, National Rural Electric Cooperative Association } \\
\text { - Manufacturer: Howard Self, ABB }\end{array}$ \\
\hline 10:45 am & BREAK \\
\hline II:00 am & $\begin{array}{l}\text { BREAKOUT SESSION I: CHALLENGES FOR INTEROPERABILITY T\&C } \\
\text { - Overview of Interoperability Standards Landscape and T\&C } \sim \text { Cuong Nguyen, NIST } \\
\text { - Facilitated Discussion: Why isn't T\&C currently working for interoperability at the needed levels? Why } \\
\text { aren't T\&C components integrated in more interoperability standards? }\end{array}$ \\
\hline $12: 15 \mathrm{am}$ & LUNCH (ON YOUR OWN) \\
\hline I:30 pm & 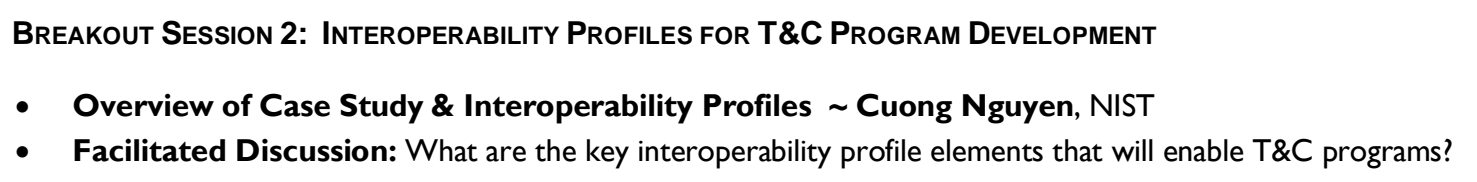 \\
\hline 2:45 pm & BREAK \\
\hline 3:00 pm & $\begin{array}{l}\text { BREAKOUT SESSION 3: PRIORITY INTERFACES FOR T\&C PROGRAM DEVELOPMENT } \\
\text { - Communications Pathway Scenarios and Interfaces Avi Gopstein, NIST } \\
\text { - Facilitated Discussion: } \\
\text { - Given the communication scenarios, what critical interfaces do we want to see T\&C form around? } \\
\text { Consider interfaces between customer assets within the domain; customer or third-party assets across } \\
\text { domains; inter-domain observability to/from utility; inter-domain controllability to/from utility; utility } \\
\text { devices across domains; and others. } \\
\text { - What will encourage/enable development/buy-in for an interoperability T\&C program? Should } \\
\text { T\&C/standards be incorporated into regulation or procurement language? Is specificity sufficient or do we } \\
\text { need other drivers? Are market conditions creating challenges for T\&C? }\end{array}$ \\
\hline $4: 30 \mathrm{pm}$ & Report Outs and Next Steps \\
\hline $5: 00 \mathrm{pm}$ & ADJOURN \\
\hline
\end{tabular}




\section{Appendix C: Background Materials}

- Interoperability Profile White Paper (Draft)

- Presentations

Background materials are available at the NIST Event Site:

https://www.nist.gov/news-events/events/2018/07/testing-and-certification-workshop 


\section{Appendix D: Acronyms}

$\begin{array}{ll}\text { AMI } & \text { Advanced metering infrastructure } \\ \text { ANSI } & \text { American National Standards Institute } \\ \text { CPS } & \text { Cyber-physical systems } \\ \text { DSP } & \text { Digital signal processing } \\ \text { EPRI } & \text { Electric Power Research Institute } \\ \text { EV } & \text { Electric vehicle } \\ \text { FSGIM } & \text { Facility Smart Grid Information Model } \\ \text { FERC } & \text { Federal Energy Regulatory Commissions } \\ \text { IEC } & \text { International Electrotechnical Commission } \\ \text { IEEE } & \text { Institute of Electrical and Electronics Engineers } \\ \text { IoT } & \text { Internet of Things } \\ \text { IIoT } & \text { Industrial Internet of Things } \\ \text { ISO } & \text { International Standards Organization } \\ \text { MH2 } & \text { Mobile host protocol } 2 \\ \text { NIST } & \text { National Institute of Standards and Technology } \\ \text { OSI } & \text { Open Systems Interconnection } \\ \text { OWL } & \text { Web ontology language } \\ \text { PLC } & \text { Programmable logic controller } \\ \text { PSC } & \text { Public Service Commission } \\ \text { PUC } & \text { Public Utility Commission } \\ \text { RF } & \text { Radio frequency } \\ \text { RFP } & \text { Request for proposal } \\ \text { SDR } & \text { Software defined radio } \\ \text { SEPA } & \text { Smart Electric Power Alliance } \\ \text { T\&C } & \text { Testing and Certification } \\ \text { USB } & \text { Universal Serial Bus } \\ & \end{array}$

